\title{
L'équivoque dans les métiers de la communication
}

\section{Bernard Dagenais et Florian Sauvageau}

\section{OpenEdition}

Journals

Édition électronique

URL : http://journals.openedition.org/communicationorganisation/1803

DOI : 10.4000/communicationorganisation. 1803

ISSN : 1775-3546

Éditeur

Presses universitaires de Bordeaux

Édition imprimée

Date de publication : 1 novembre 1995

ISSN : 1168-5549

\section{Référence électronique}

Bernard Dagenais et Florian Sauvageau, «L'équivoque dans les métiers de la communication », Communication et organisation [En ligne], 8| 1995, mis en ligne le 26 mars 2012, consulté le 19 avril 2019. URL : http://journals.openedition.org/communicationorganisation/1803 ; DOI : 10.4000/ communicationorganisation. 1803

Ce document a été généré automatiquement le 19 avril 2019

(c) Presses universitaires de Bordeaux 


\title{
L'équivoque dans les métiers de la communication
}

\author{
Bernard Dagenais et Florian Sauvageau
}

\section{NOTE DE L'AUTEUR}

Ce texte reprend des éléments du chapitre des auteurs dans l'ouvrage Communication et développement international, préparé sous la direction de Thérèse Paquet-Sévigny et publié aux Presses de l'Université du Québec, en 1996.

1 Depuis le début des années 1990, de nombreux analystes ont constaté le flou des concepts d'information et de communication (Bougnoux 1995) et dénoncé la confusion qui entoure les métiers de la communication. De façon plus spécifique, d'autres se sont inquiétés de la montée de la communication institutionnelle et des dangers qu'elle fait courir au journalisme. En France, Tixier-Guichard et Chaize (1993) ont sonné l'alarme avec leur ouvrage Les Dircoms. Pour eux, informer correctement est devenu plus difficile que jamais, «parce que toutes les institutions (politiques, économiques, sociales ou culturelles) se sont dotées de "directions de la communication " ayant pour mission de produire un discours flatteur et élogieux à leur égard. Cette habileté trompe les médias » (TixierGuichard et Chaize 1994, p. 28).

\section{Journalistes et relationnistes : frères ennemis}

2 Au Québec, il y a des années que journalistes et relationnistes, les frères ennemis du monde de la communication, entretiennent des rapports difficiles. Dès les années 1960, la création de services d'information au gouvernement du Québec avait provoqué quelque inquiétude chez certains correspondants parlementaires. En 1968, le désir de plusieurs anciens journalistes, devenus agents d'information, d'adhérer à la Fédération professionnelle des journalistes nouvellement créée, et le rejet de leur demande, avait 
conduit à des débats déchirants entre ex-collègues. Les agents d'information plaidaient qu'ils devaient au sein de l'administration publique servir l'intérêt public tout autant que le font les journalistes.

Déjà, la confusion s'installait dans les métiers de la communication. Presque dix ans plus tard, l'ambiguïté persistait. En préfaçant les Actes d'un colloque justement intitulé Agent d'information: agent de confusion?, le relationniste Serge de la Rochelle (1977, p. vIII) avait perçu la nécessité de préciser qu'un agent d'information n'était ni un animateur social, ni un analyste politique, ni un journaliste, mais un "professionnel qui, librement et volontairement, a décidé de mettre sa compétence et son talent au service d'une organisation qui le rémunère pour son travail ».

Depuis, à la faveur de la création de services d'information et de communication dans la plupart des secteurs d'activités, les attaques réciproques entre journalistes et relationnistes se sont multipliées et les débats sont devenus plus acrimonieux. Le journaliste Rodolphe Morissette a posé en 1983 les paramètres du débat, comme bien des journalistes le perçoivent encore aujourd'hui :

«Par leur nombre, par le bruit qu'ils font, par l'espace et le temps qu'ils dévorent maintenant dans la presse quotidienne, par journalistes interposés, les relationnistes professionnels définissent maintenant «l'actualité » qui est jour après jour au programme et ce que sont les «nouvelles ».

« Encombrer la presse, l'encadrer, la forcer à traiter quotidiennement de ce qu'on veut qu'elle traite et, surtout, la distraire de parler de ce dont on ne veut pas qu'elle parle ».

5 Ce discours correspond tout à fait à la « théorie » journalistique de la méfiance nécessaire vis-à-vis des sources d'information: «les sources (et leurs "mercenaires», les relationnistes) ont une image à construire ou à entretenir, des intérêts à défendre, quelque chose à cacher » (Sauvageau 1991, p. XIV).

Des relationnistes répliquent qu'ils ne sont pas la pour manipuler la presse, «mais bien pour la forcer à aller au-delà, à mieux regarder des deux côtés de la médaille, pour tenir compte de tous les points de vue, bref, à exercer un jugement journalistique plus mûri » (Francoeur 1984). Celui qui tient ces propos, Luc Beauregard, un ancien journaliste devenu PDG du plus important cabinet de relations publiques au Québec, se demande aussi pourquoi les relationnistes n'auraient pas le droit, par delà les journalistes-juges, ce qu'il appelle "La Cour d'information », de soumettre leur point de vue au tribunal de l'opinion publique. Et il invite ensuite journalistes et relationnistes à la collaboration "pour assurer une plus libre circulation de l'information». Cette invitation heurte évidemment de plein fouet la thèse de la «source dont il faut se méfier » à laquelle adhèrent toujours de nombreux journalistes.

7 Les auteurs du présent article croient que le problème est plus complexe que ne le laissent généralement croire journalistes et relationnistes et que les admonestations réciproques ne mènent nulle part. Ils ne s'entendent toutefois par sur tout. L'un (Sauvageau) a pendant des années, pratiqué le journalisme, l'autre les relations publiques (Dagenais). Leur approche est forcément différente. L'un considère que la montée des relationnistes a transformé le journalisme pour en faire un métier strictement passif : les médias, plutôt que de chercher la nouvelle, se satisfont de faire le tri dans la masse des informations reçues des diverses organisations. L'autre pense que le pouvoir immense que détiennent les médias sur nos façons d'être, de faire et de penser, jouxté à la logique et ç la routine journalistiques ont poussé les diverses institutions à se défendre et à 
s'organiser. Ils s'entendent toutefois sur un point essentiel. Les deux métiers baignent dans une confusion qu'occultent les discours des « frères ennemis » de la communication. Alors que les finalités du journalisme et des relations publiques sont différentes, voire irréconciliables : en principe, le journaliste agit dans l'intérêt général, le relationniste sert des intérêts particuliers, ceux de l'organisation qui l'emploie.

8 À partir de nombreux exemples et d'observations personnelles, les auteurs du présent article souhaitent montrer qu'au-delà de dérapages occasionnels et des discours, de nombreuses ambiguïtés existent entre les métiers de journalistes et de relationnistes, bien qu'ils vivent dans des univers opposés par vocation première. Cette confusion des genres dans les métiers de la communication ne facilite ni le traitement, ni la circulation adéquate des informations et constitue l'un des enjeux principaux des discussions qui ont cours sur le flou de la communication.

\section{La confusion des genres}

\section{Confusion dans le statut}

Il n'existe pas de définition propre du métier de journaliste ou de relationniste. Même si les praticiens de l'un et l'autre métier ont encadré leur profession de principes distincts et bien définis, les deux métiers sont en pratique complètement livrés à eux-mêmes, avec les avantages d'une grande liberté... et les inconvénients d'un abus de cette liberté.

Bien sûr, le fait d'être membre d'une fédération professionnelle de journalistes ou de posséder la carte de presse d'une part, ou de faire partie d'une société de relationnistes ou d'une association des attachés de presse et de communication d'autre part, signifie que l'on répond à certains critères d'admission mis en avant par ces organisations; mais cela ne constitue aucun frein à pratiquer sans ces attributs ces mêmes métiers, pour quiconque ne répondrait pas aux critères, dans la grande majorité des pays occidentaux du moins.

11 Résultat: alors que les journalistes accusent les relationnistes de vicier l'information, alors que les relationnistes se désolent du pouvoir abusif des médias, voilà que les deux métiers opposés se rejoignent dans une même confusion. La frontière entre les deux professions devient si ténue qu'on ne voit plus toujours la distance qui les sépare. Essayons de mieux cerner quelques éléments qui expliquent cette confusion grandissante.

12 L'absence de formation spécifique - Le monde du journalisme et celui des relations publiques n'ont pas de filière exclusive de formation. Les deux professions résistent à toute initiative qui obligerait les uns ou les autres à passer à travers un moule unique. C'est, estime-t-on, la diversité des formations et des expériences qui crée justement l'excellence de ces métiers.

13 Paradoxalement, ce refus d'une formation spécifique a conduit à la mise en place de programmes hybrides, où les uns et les autres peuvent recevoir des formations ou des spécialisations identiques. Au Québec, si certains revendiquent des écoles distinctes et bien identifiées pour la préparation professionnelle des journalistes et des relationnistes, la formation universitaire se donne encore dans les mêmes facultés et les étudiants sont le plus souvent appelés à se former à un même tronc commun. S'il existe en France des écoles de journalisme, de relations publiques ou d'attachés de presse reconnues, de nombreux professionnels des deux champs de pratique sont aussi formés dans les 
universités où le nombre d'étudiants (et de programmes) en « info-com » s'est développé de façon quasi exponentielle au cours des années 1980. Palmer (1991) reconnaît que dans la majorité des cas, les universités forment des "généralistes-spécialistes» en « information-communication ». La même ambiguïté se retrouve aux États-Unis où, dans plusieurs écoles de journalisme, on enseigne souvent tout autant la publicité que les relations publiques.

L'accès à ces professions est ouvert à tous - Compte tenu qu'il n'y a aucune exigence de formation spécifique, les deux professions sont donc accessibles à tout le monde. Apparait ainsi, chaque année, un nouveau contingent d'individus venus d'horizons divers pour acquérir les rudiments de base et comprendre les complexités des métiers de la communication en les pratiquant. Cette liberté d'accès permet d'ouvrir ces métiers à toutes les compétences et d'enrichir leur portée par la diversité des points de vue que cette ouverture alimente. Mais cela les rend également accessibles à toutes les médiocrités.

Apparaissent de même, dans cet univers des Communications, des individus propulsés au rang de stars par la magie des cotes d'écoute. Désormais des personnalités du monde politique, des arts ou du sport, par exemple, peuvent se dire du jour au lendemain journalistes ou relationnistes, ou l'un et l'autre en même temps. Ces personnalités vont parfois apporter une perception extrêmement éclairante sur leur ancien métier. Mais la confusion peut devenir totale lorsque le même individu, par l'expertise que peut lui conférer son métier d'origine, parle avantageusement, en tant que «journaliste », d'un produit qu'il utilise, pour devenir ensuite le porte-parole officiel du même produit, dans des messages publicitaires; ou, à l'inverse, lorsqu'une personne associée à un produit devient ensuite chroniqueur spécialisé dans un média, avec mission d'analyser «objectivement» les avantages de ce même produit (le mot "produit» doit ici être compris dans son sens le plus large: automobile, forfait-voyage, spectacle, équipe sportive, ou parti politique). On a même vu des cas où le " journaliste » vedette était aussi actionnaire de l'entreprise fabriquant le produit en question. Il ne s'agit pas la d'une règle générale, bien sûr, mais d'un effet pervers, inévitable peut-être, dans un secteur d'activité sans aucune balise qui permet aux mêmes individus de pratiquer en même temps des métiers opposés quant à leur finalité.

La grande fluidité entre les deux métiers - Le président de la plus grande agence de relations publiques au Québec est un ancien journaliste. D'ailleurs, plusieurs agences ont été créées par des journalistes, ou embauchent d'anciens journalistes, afin de tirer profit de leur bonne connaissance des médias. Dès les années 1960, comme on l'a vu, plusieurs journalistes attirés par des conditions de travail plus intéressantes, avaient accepté des postes d'agents d'information dans le secteur public. C'est aussi dans le bassin des journalistes que puiseront souvent les politiciens, lorsqu'ils cherchent à adjoindre un attaché de presse à leur cabinet politique. Plusieurs journalistes deviennent ainsi, à l'occasion ou de façon permanente, des relationnistes ou des attachés de presse. Certains d'entre eux reviennent ensuite au journalisme.

La situation est plus confuse encore dans le cas des pigistes, dont bon nombre doivent alterner sans cesse d'un genre à l'autre, selon les contrats. La grande précarité de leur situation professionnelle ne leur permet pas toujours de refuser les mandats les plus lucratifs, au nom de principes éthiques. Ils le font d'autant moins que l'exemple est généralisé. Pour arrondir les fins de mois, plusieurs journalistes, même ceux qui jouissent d'une certaine sécurité d'embauche, pratiquent ce qu'on appelle "des ménages». Ils 
acceptent des contrats de consultation pour des entreprises ou des organisations qu'ils pourraient même être appelés à couvrir. Tixier-Guichard et Chaize (1993, p. 509) et Brugidou (1993) citent l'exemple d'un journaliste du Monde, appelé à couvrir l'affaire du sang contaminé alors qu'il avait été auparavant contractuel pour les accusés. Gunther (1994) cite le cas d'un producteur d'une émission d'information d'une grande chaîne de télévision américaine qui a servi de conseiller en communication de Bill Clinton lors des élections présidentielles de 1993. Selon une enquête d'Impact-Recherche (1988) auprès des journalistes québécois, $40 \%$ des journalistes interrogés ont dit arrondir ainsi leurs fins de mois en effectuant des tâches reliées à la publicité et aux relations publiques.

Des journalistes réalisent aussi des biographies de personnalités politiques ou des portraits d'entreprise. Le phénomène est tellement commun que personne ne s'étonne de voir ces livres signés du nom d'un journaliste. Certains de ces ouvrages manifestent une rigueur à toute épreuve. Par contre, d'autres sont soit complaisants, soit violemment critiques. Dans un cas comme dans l'autre, elles ne relèvent alors pas toujours d'une enquête rigoureuse, mais plutôt d'une attitude complice ou d'un règlement de compte. Faut-il s'étonner de constater que ceux qui ont été plus complaisants puissent obtenir un jour quelque sinécure?

19 Tous ces éléments jettent une confusion certaine entre deux métiers qui, en théorie, ont dressé entre eux des frontières infranchissables... pourtant allègrement franchies.

\section{Confusion sémantique}

Les termes mêmes de journaliste et de relationniste sont utilisés de façon confuse. Dans certains pays, on utilise le terme « journaliste d'entreprise » pour désigner les rédacteurs de journaux d'entreprises ou d'institutions, rédacteurs qui se sont faits reconnaître un statut légal, à titre de journalistes spécialisés. En France, ils sont regroupés dans l'« Union des journaux et des journalistes d'entreprise » et peuvent être détenteurs d'une carte de presse officielle, distincte toutefois de celle des journalistes professionnels de la presse. On y retrouvera dans bien des cas les rédacteurs attitrés du service des Communications, qui sont pourtant clairement des relationnistes. Ainsi le même titre professionnel est utilisé pour désigner des gens qui tentent de valoriser l'image de l'entreprise ou organisation qui les embauche, et par des gens qui recherchent en principe les faits et la vérité, au service du public.

21 Par ailleurs, avouons-le, des «journalistes» travaillant dans des médias dits « d'information » exercent un rôle qui s'apparente davantage à celui d'attaché de presse ou d'agent de promotion que de journaliste. Ce genre de confusion existe en particulier (mais pas exclusivement) dans certaines sections spécialisées des journaux, comme le journalisme de voyage, le journalisme de mode, voire le journalisme sportif, où la frontière entre l'information critique et la promotion des produits offerts est difficile à cerner.

À l'opposé, certains rédacteurs de journaux institutionnels disposent d'une grande marge de manœuvre. Parlant, pour la France, de « ceux qui réalisent les magazines des villes, des départements ou des régions » Charon (1993, p. 110-111) signale par exemple que, « dans l'exercice de leur activité, ces rédacteurs réaliseront des enquêtes, des reportages, des interviews. Ils rédigeront aussi bien des brèves, des billets que des papiers de fond. (...) Quant à la liberté de ton et à l'autonomie rédactionnelle, elles seront bien souvent équivalentes, sinon supérieures, à celles des hebdomadaires et des quotidiens 
régionaux ». Au Québec, le même constat peut être fait dans le cas de certains magazines publiés par des ministères ou organismes gouvernementaux ou para-publics. Ainsi, l'office (gouvernemental) de la protection du consommateur publie Protégez-vous, un magazine dont les enquêtes et les analyses de divers produits sont régulièrement reprises par les médias et auquel peuvent contribuer, à la pige, certains journalistes qui collaborent aussi avec des entreprises de presse. Ces derniers sont-ils moins journalistes et davantage agents d'information ou « relationnistes » lorsqu'ils écrivent dans Protégezvous?

23 Il existe par ailleurs un autre exemple de cette confusion sémantique entre les deux métiers de la communication: c'est l'utilisation de l'expression "affaires publiques ». Dans le monde du journalisme, les affaires publiques désignent les émissions qui traitent de la dimension socio-politique de la société, des affaires de la Cité. Dans l'univers des entreprises, les affaires publiques désignent, par euphémisme, toute tentative d'influencer les enjeux publics. On qualifie de plus en plus souvent la vice-présidence ou la direction qui gère les Communications de «direction des affaires publiques ». On y inclut les activités de lobby, les activités communautaires et toute initiative de nature à valoriser l'image institutionnelle de l'entreprise. En fait, c'est la dimension sociopolitique des relations de l'entreprise avec ses différents publics qui est ainsi désignée.

\section{Confusion des taches}

Définir les taches d'un journaliste ou d'un relationniste consiste à dresser une nomenclature complexe d'activités et d'actions non seulement diversifiées, mais parfois contradictoires. Si le grand journaliste d'un média important peut revendiquer une autonomie absolue de travail, le rédacteur-vendeur de publicité d'un hebdomadaire régional est emprisonné dans une perpétuelle schizophrénie : il doit protéger par intérêt économique ce qu'il souhaiterait condamner par souci éthique et responsabilité sociale.

Le monde varié du journalisme - Dans ses études sur le statut du journaliste, Derieux $(1988,1991)$ constate le vague qui entoure le métier de journaliste. Qu'y a-t-il de commun, demande-t-il, entre l'éditorialiste d'un grand quotidien national, Ie présentateur du journal télévisé, la rédactrice d'un journal de mode, le critique gastronomique d'une publication spécialisée, etc.

Pourtant, ce sont tous des « journalistes ». Et Derieux conclut : «On ne peut se satisfaire véritablement de la situation présente qui, pratiquement partout, aboutit à englober en une seule et même appellation (...) des professionnels dont les activités (...) comportent probablement davantage de différences que de ressemblances » (Derieux 1988, p. 3).

Entre les animateurs vedettes d'horizons divers pratiquant avec succès l'art de l'infotainment, les chroniqueurs sportifs complètement inféodés à leur équipe professionnelle locale, les chroniqueurs du tourisme vivant au crochet des commanditaires, les chroniqueurs politiques déjeunant avec les grands de ce monde et les reporters affectés aux faits divers, se développent des formes de journalisme fort éloignées. " Pour dire qui sont les journalistes, encore faudrait-il définir le journalisme ", précise Ruellan (1993a, p. 211). «Et la, autant en faire son deuil. Car dès l'origine, le journalisme est un incroyable méli-mélo de fonctions bien différentes». Ce flou n'embarrasse pas Ruellan qui considère que la fluidité du journalisme, un métier fondamentalement perméable, en constitue sa force : « elle offre au métier des capacités d'amalgame et de renouvellement permanent qui en font une perpétuelle profession 
nouvelle; le flou de sa constitution permet aussi d'offrir moins de prises extérieures sur le groupe des praticiens, qui se révèle difficile à contraindre » (Ruellan 1993b, p. 224).

Le métier que pratique le journaliste peut aussi se confondre directement avec d'autres métiers de la communication. Lors du colloque organisé par la Fédération nationale des Communications du Québec (1990) sur le thème de La publicité dans l'information, des participants ont donné des exemples précis de la tendance à convertir l'information en objet de publicité ou de marketing. Falardeau (p. 8) cite l'anecdote d'un journaliste qui trouvait plus valorisant de rédiger des publi-reportages pour lesquels il recevait des félicitations que de vrais reportages qui étaient critiqués parce que trop engagés. Sauvageau (p. 27) décrit le geste d'un nouveau patron de réseau de télévision canadien qui a décidé que ses bulletins de nouvelles devaient répondre aux règles du marketing commercial.

Le monde éclaté des relations publiques - On les nomme relationnistes, agents d'information, attachés de presse, chargés de la communication, communicateurs d'entreprise, responsables des affaires publiques ou communautaires. Ils sont rédacteurs ou éditeurs de publications, ils font des relations de presse, du placement publicitaire, ils organisent des colloques ou des expositions, ils réalisent des documents audio-visuels. Ils répondent aux demandes de renseignements ou aux plaintes des citoyens consommateurs. Ils diffusent de l'information auprès des employés. Ils accueillent les visiteurs, gèrent les crises internes ou externes de l'entreprise, et font connaître les coups d'excellence. Ils font du lobby et gèrent parfois des budgets de représentation ou de commandite. En fait, ils occupent cent métiers différents. Certains sont chargés de diffuser des informations factuelles et aussi objectives que des horaires. D'autres sont payés pour construire l'image d'une entreprise, d'un individu, d'une cause et sont appelés à manipuler la perception de leur "produit " auprès d'une clientèle cible. Certains pratiquent le grand reportage et $y$ chantent les bienfaits de leur entreprise.

30 Il existe donc, chez les relationnistes aussi, une immense disparité des tâches, certaines proches des idéaux du journalisme, d'autres aussi éloignées que le sont les plus condamnables égarements de la presse.

31 La confusion croissante des mandats - Quelle différence y a-t-il entre une entreprise commerciale qui parraine et subventionne une activité culturelle, et un média d'information qui commandite le même événement? Ils font tous deux des relations publiques. De même, lorsque des journalistes accordent leur soutien à des causes qu'ils souhaitent défendre, prêtent leur concours et leur prestige à des événements, ils sont davantage des « relationnistes » que des journalistes.

Au début de l'année 1996, au Québec, le quotidien Le Devoir s'est associé à l'entreprise publique d'hydro-électricité, Hydro-Québec, commanditaire d'un concours de journalisme organisé par le journal et dont le gagnant deviendra pour une année journaliste stagiaire en matière d'environnement. Vu sous l'angle de relations publiques et de l'image, le journal et Hydro-Québec y trouvent leur compte. Le Devoir manifeste son intérêt pour les aspirants journalistes qui ne trouvent pas d'emploi. Hydro-Québec montre qu'elle se préoccupe des questions d'environnement. Dans une perspective de journalisme autonome, les choses sont différentes. Les journalistes du quotidien ont reproché à leur direction d'avoir mis en danger la crédibilité du journal. L'éthique « n'est pas froissée » a répliqué la directrice : "Hydro-Québec commandite le prix et c'est tout». Le Devoir est responsable du déroulement du concours, et de l'encadrement du stagiaire. L'affaire se 
comprend mieux quand on sait que le président de la Fondation du Devoir était auparavant vice-président (Communications et Relations publiques) d'Hydro-Québec.

Lorsque des entreprises ou organisations pratiquent ce qu'on appelle la publicité de plaidoyer - advocacy advertising -, elles utilisent le genre éditorial d'un média. Puisque les journalistes ne « répètent » pas bien les propos des entreprises, celles-ci vont acheter des espaces publicitaires pour s'assurer que leurs idées sont diffusées la et où elles le souhaitent. C'est ainsi qu'en Amérique, on a vu les grandes entreprises réserver de pleines pages de publicité pour justifier leurs pratiques commerciales: les pétrolières et les banques ont eu recours à cette pratique lorsqu'elles furent accusées de profiter des crises qui touchaient leur secteur d'activité. Les syndicats vont utiliser le même procédé pour condamner l'attitude des patrons pendant un conflit ouvrier. Désormais, par la publicité, les organisations vont partager la fonction éditoriale des médias, c'est-à-dire qu'elles vont essayer d'orienter la façon de percevoir la réalité.

Désormais, entre les relations publiques et le journalisme, il y a autant de complicité que d'antagonisme; les taches à réaliser sont parfois identiques, parfois irréconciliables; les mandats qu'ils assument se ressemblent ou s'opposent. L'ambiguïté des rôles obscurcit les contours de chacun des deux métiers, théoriquement si différents.

\section{Une ambiguïté qui s'accentue}

Retranchés derrière leurs positions traditionnelles, journalistes et relationnistes continuent le plus souvent de définir l'Autre comme l'ennemi. Ils occultent ainsi la complexité du problème et rendent plus ardue la recherche d'une solution, au moment où de nouveaux éléments viennent accroître la confusion.

\section{La formation universitaire}

Nous avons déjà soulevé l'absence, en Amérique du Nord en particulier, de formation spécifique au journalisme et aux relations publiques. C'est après un cursus commun que l'apprenti communicateur orientera son choix de travail en fonction parfois de ses goûts, plus souvent du marché du travail. Or le marché du travail favorise nettement les relationnistes. Dans les médias, on réduit le personnel, et on « rationalise » les opérations de collecte de l'information. D'autre part, l'univers de la communication d'entreprise se développe de façon continuelle. Les universités, à la recherche de clientèle additionnelle, vont favoriser ce secteur qui jouit de l'engouement de la population étudiante.

D'où la tentation de regrouper ou de maintenir dans un même centre la formation aux divers métiers de la communication, d'autant que les techniques du journalisme sont celles qu'utilisera le communicateur d'entreprise pour réaliser ses communiqués-vidéo, ses documentaires et autres activités de communication, même si les finalités sont différentes de celles du journalisme.

Pour le relationniste Serge de la Rochelle (1977, p. IX), « Former des relationnistes n'a rien à voir avec la formation qu'on veut donner aux journalistes. Les objectifs des uns et des autres diffèrent considérablement. Le temps est venu, je pense, de briser avec cette tradition qui se retrouve dans plusieurs universités nord-américaines et de cesser cette domination qu'exercent les écoles de journalisme sur l'enseignement des relations publiques ». C'était en 1977. Quelque 20 ans plus tard, la proposition s'est inversée. Ce 
sont les journalistes qui ont été happés par la dynamique nouvelle de la communication d'entreprise et qui tentent d'échapper à son emprise. Mais dans tous les cas, la confusion règne.

\section{Les changements de rôle}

39 Nous posions au début qu'en principe le journaliste agissait dans l'intérêt général et que le relationniste servait des intérêts particuliers. La réalité nous oblige à repenser cette assertion. Car de plus en plus, les journalistes sont soumis aux lois du marché et leur couverture de la réalité est dictée par une logique trop souvent uniquement économique. Les services de publicité des médias imposent des règles du jeu (Demers 1991) qui nous ramènent à une époque que l'on croyait révolue : la page payée et la page donnée et le publi-reportage devenu «l'infomercial». Désormais, la logique organisationnelle des médias et les intérêts commerciaux de ceux-ci orientent le choix et l'espace consacrés à chaque élément de l'activité humaine. Les journalistes doivent subir ces choix (Bogart 1995, Squires 1993).

D'un autre côté, les entreprises privées et autres organisations qui ne pensent a priori qu'à leur profit et intérêt ont compris qu'il était essentiel pour leur image qu'elles s'engagent davantage dans les causes sociales et humanitaires. Ces nouveaux rôles qu'occupent les partenaires sociaux jettent encore plus de confusion dans les métiers de la communication : celui qui devait s'intéresser à l'intérêt général est trop souvent soumis aux intérêts privés des bailleurs de fonds ; celui qui doit travailler pour l'intérêt privé de son employeur est tout à coup investi d'une mission publique.

\section{L'éthique}

41 Face à ces changements, nombreux sont ceux qui en appellent à l'éthique et réclament une nouvelle déontologie pour les métiers de la communication. La suggestion n'a pourtant rien d'original. Journalistes et relationnistes débattent depuis des années la pertinence d'adopter des règes de déontologie. Les difficultés que crée l'élaboration de règles acceptées par tous ne peuvent toutefois pas être invoquées pour justifier l'absence d'une réflexion constante sur la pratique des métiers de la communication et sur la confusion qui l'entoure.

D'ailleurs, cette réflexion existe. Dans les revues spécialisées dans lesquelles les journalistes, les relationnistes et les autres communicateurs parient de leur métier, ils font preuve de beaucoup de lucidité et n'hésitent pas à analyser et à condamner des situations jugées inacceptables, situations que plusieurs d'entre eux acceptent pourtant dans leur pratique quotidienne. Mais ces critiques sortent rarement du cercle des initiés et toujours, l'Autre reste l'ennemi.

L'équivoque des métiers de la communication, au double plan de la confusion des genres et des abus qu'elle engendre, jette encore plus d'incertitude sur la libre circulation de l'information et des idées et sur le contrôle des enjeux socio-politiques. 


\section{BIBLIOGRAPHIE}

BOGART Leo 1995, Commercial Culture : the media system and the public interest, Oxford University Press, $384 \mathrm{p}$.

BOUGNOUX, Daniel 1995, La communication contre l'information, Hachette, 143 p.

BRUGIDOU Mathieu 1993, "L'affaire du sang contaminé : la construction de l'événement dans Le Monde» -in la revue Mots/Les langages du politique, sur le thème « Rhétoriques du journalisme politique ", n³7, déc. 1993, pp. 29-46.

CHARON Jean-Marie 1993, Cartes de presse : enquête sur les journalistes, Stock, 356 p.

DE LA ROCHELLE Serge 1977, Agent d'information : agent de confusion? Groupe de recherche en information et communication, Département d'information et de communication, Université Laval, 216 p. + annexe.

DEMERS François (1991), « Du côté des journalistes et de « leurs » messages : le cas de l'information et de la publicité dans les années quatre-vingt », in Beauchamp Michel (dir), Communication publique et société : repères pour la réflexion et l'action, Boucherville, Gaëtan Morin, pp. 217-245.

DERIEUX Emmanuel 1988, « Journalistes », Répertoire de droit du travail, Paris, Dalloz, 30 avril.

DERIEUX Emmanuel 1991, Manuel de droit de la communication, Paris, Librairie générale de droit et de jurisprudence, $600 \mathrm{p}$.

FEDERATION NATIONALE DES COMMUNICATIONS 1990, La publicité dans l'information : l'invasion tranquille, Actes du colloque tenu les 14 et 15 juin 1990 à Québec, 170 p.

FRANCOEUR L.G. 1984, « Journaliste et relationniste doivent agir en professionnel », Le 30, vol. 8, $\mathrm{n}^{\circ} 3$, mars, p. 12 ss.

GUNTHER Marc, 1994, « A Clinton friend on media spot », The Philadelphia Inquirer, 10 février 1994, p. F1-F12.

IMPACT-RECHERCHE 1988, « Enquête auprès des journalistes québécois », Rapport présenté à la Fédération Professionnelle des Journalistes du Québec, novembre, $43 \mathrm{p}$.

MORISSETTE R. « La presse quotidienne est en train de se faire avoir », Le 30, vol. 7, n 10, décembre 1983, p. 14 ss.

PALMER Michael, 1991, «La difficile définition des filières françaises - La formation ", in L'État des médias, sous la direction de Jean-Marie Charron, avec la collaboration de Florian Sauvageau, Boréal - La Découverte -Médiaspouvoirs - CFPJ, pp. 418-419.

RUELLAN Denis 1993a, « Les frontières d'une vocation », in Jean-François Lacan, Michael Palmer, et Denis Ruelland, Les journalistes : stars, scribes et scribouillards, Paris, Syros, pp. 207-273.

RUELLAN Denis, 1993b, Le professionnalisme du flou, Presses de l'Université de Grenoble, 240 p. SAUVAGEAU Florian, 1991, «Introduction », in Jean Charron, Jacques Lemieux Jacques et Florian Sauvageau, Les journalistes, les médias et leurs sources, Boucherville, Gaëtan Morin, 237 p. 
SQUIRES James D. 1993, Read All About It. The Corporate Takeover of America's Newspaper, New York

Times Book, $244 \mathrm{p}$.

TIXIER-GUICHARD Robert et Daniel CHAIZE, 1993, Les dircoms. A quoi sert la communication?, Seuil, $608 \mathrm{p}$.

TIXIER-GUICHARD Robert et Daniel CHAIZE 1994, « Les médias à l'ère du mensonge : La communication contre l'information », Le Monde Diplomatique, avril 1994, pp. 28-29.

\section{RÉSUMÉS}

Lorsque l'on aborde le couple information-communication, la tendance la plus répandue est d'opposer les deux termes. Cette attitude est principalement celle des journalistes qui s'estiment agressés par les professionnels de la communication qui prétendent leur souffler, aux deux sens du terme, une partie de plus en plus grande de leur travail. La confusion règne donc dans les métiers de l'information et de la communication, entretenue par les discours des uns et des autres. Un journaliste et un «relationniste» analysent ici, à deux voix, les ambigüités qui fondent cette confusion.

When we tackle the twin topics information and communication the most common tendency is to oppose the two. This attitude is mainly that of journalists who feel threatened by communication professionals who might steal or demolish more and more of the work that they do. There is therefore a great deal of confusion in the information and communication professions which is fuelled by both sides. Here, a journalist and a public relation manager » both analyse the ambiguities which are behind this confusion.

\section{AUTEURS}

\section{BERNARD DAGENAIS}

Bernard Dagenais possède une maîtrise et un doctorat du Centre d'Études des Communications de Masse de l'École pratique des Hautes Études en Sciences Sociales de Paris. II est professeur agrégé au Département d'information et de communication de l'Université Laval à Québec et consultant en communication aux niveaux national et international depuis 1986 après avoir œuvré pendant 10 ans comme cadre supérieur dans différentes Directions de communication du Gouvernement du Québec. Bernard Dagenais est autant un praticien qu'un théoricien de la communication publique. Ses champs de recherche se sont concentrés autour de deux thèmes principaux : les Communications institutionnelles des organisations qui veulent affirmer leur présence sur la scène publique ; et le pouvoir des médias qui contrôlent la scène publique et qui imposent aux organisations une routine et une logique particulières. Ces deux thèmes s'articulent dans la relation particulière qui s'instaure entre relationnistes et journalistes.

\section{FLORIAN SAUVAGEAU}

Avocat et journaliste, Florian Sauvageau est professeur titulaire au Département d'information et de communication de l'Université Laval à Québec. Il a animé de nombreuses émissions à la radio et à la télévision de Radio-Canada et a collaboré à la réalisation et à la scénarisation de plusieurs films documentaires pour l'Office National du Film (ONF). Il a aussi été directeur de l'information et directeur de la rédaction au 
quotidien Le Soleil. Co-auteur de Droit et journalisme au Québec. il a publié plusieurs articles et co-dirigé la publication d'ouvrages consacrés aux médias, au journalisme et à la liberté et à la responsabilité de la presse. En 1985-1986, il a été co-président du Groupe de travail sur la politique de la radiodiffusion au Canada (Caplan-Sauvageau). Florian Sauvageau est aussi directeur du Centre d'Études sur les Médias de l'Université Laval. 